\title{
Article
}

\section{Possibility Pythagorean bipolar fuzzy soft sets and its application}

\author{
M. Palanikumar ${ }^{1, *}$ and K. Arulmozhi ${ }^{1}$ \\ 1 Department of Mathematics, Annamalai University, India. \\ * Correspondence: palanimaths86@email.com; Tel.: +919976931386 \\ Communicated by: Muhammad Kamran Jamil \\ Received: 15 April 2021; Accepted: 28 May 2021; Published: 25 June 2021.
}

\begin{abstract}
We interact the theory of possibility Pythagorean bipolar fuzzy soft sets, possibility bipolar fuzzy soft sets and define complementation, union, intersection, AND and OR. The possibility Pythagorean bipolar fuzzy soft sets are presented as a generalization of soft sets. Notably, we tend to showed De Morgan's laws, associate laws and distributive laws that are holds in possibility Pythagorean bipolar fuzzy soft set theory. Also, we advocate an algorithm to solve the decision making problem primarily based on soft set model.
\end{abstract}

Keywords: Pythagorean bipolar fuzzy soft set; Possibility Pythagorean bipolar fuzzy soft set; Decision making problem.

MSC: 03E72; 06D72.

\section{Introduction}

$\mathbf{M}$ any uncertain theories are put forward as fuzzy set [1], intuitionistic fuzzy set [2], bipolar fuzzy sets [3] and Pythagorean fuzzy set [4]. Zadeh [1] introduced fuzzy set and suggests that decision makers can solving uncertain problems by considering membership degree. The concept of intuitionistic fuzzy set was introduced by Atanassov [2] and characterized by a degree of membership and non-membership satisfying the condition that sum of its membership degree and non membership degree do not exceeds 1 . However, we may interact a problem in decision making events where the sum of the degree of membership and non-membership of a particular attribute exceeds one. The concept of Pythagorean fuzzy sets introduced by extending the intuitionistic fuzzy sets and characterizing the condition that squares of sum of its membership and non membership degree do not exceeds 1 [4]. The theory of soft sets proposed by Molodtsov [5] is a tool of parameterization for coping with the uncertainties. In comparison with other uncertain theories, soft sets more accurately reflects the objectivity and complexity of decision making during actual situations. Moreover, the combination of soft sets with other mathematical models is also a critical research area. Maji et al., introduced the concept of fuzzy soft set and the intuitionistic fuzzy soft set in [6] and [7] respectively. Abdullah et al., [8] initiated the concept of bipolar fuzzy soft sets and Alkhazaleh et al., [9] introduced the concept of possibility fuzzy soft sets.

In 2015, Peng et al., [10] extended fuzzy soft set to Pythagorean fuzzy soft set. The purpose of this paper is to extend the concept of possibility Pythagorean fuzzy soft sets to parameterization of possibility Pythagorean bipolar fuzzy sets. We further establish a similarity measure based on soft model.

\section{Preliminaries}

Definition 1. $[4,11]$ Let $U$ be a non-empty set of the universe. The Pythagorean fuzzy set (PFS) $A$ in $U$ is an object having the form $A=\left\{x, \mu_{A}(x), \eta_{A}(x) \mid x \in U\right\}$, where $\mu_{A}(x)$ and $\eta_{A}(x)$ represent the degree of membership and degree of non-membership of $A$ respectively. Consider the mappings $\mu_{A}: U \rightarrow[0,1]$ and $\eta_{A}: U \rightarrow[0,1]$ such that $0 \leq\left(\mu_{A}(x)\right)^{2}+\left(\eta_{A}(x)\right)^{2} \leq 1$. The degree of indeterminacy is determined as $\pi_{A}(x)=\left[\sqrt{1-\left(\mu_{A}(x)\right)^{2}-\left(\eta_{A}(x)\right)^{2}}\right]$. Here $A=\left\langle\mu_{A}, \eta_{A}\right\rangle$ is called a Pythagorean fuzzy number (PFN). 
Definition 2. [12] Let $U$ be a non-empty set of the universe. The Pythagorean bipolar fuzzy set (PBFS) $A$ in $U$ is an object having the form $A=\left\{x, \mu_{A}^{+}(x), \eta_{A}^{+}(x), \mu_{A}^{-}(x), \eta_{A}^{-}(x) \mid x \in U\right\}$, where $\mu_{A}^{+}(x), \eta_{A}^{+}(x)$, $\mu_{A}^{-}(x), \eta_{A}^{-}(x)$ represent the degree of positive membership, degree of positive non-membership, degree of negative membership and degree of negative non-membership of $A$ respectively. Consider the mappings $\mu_{A}^{+}, \eta_{A}^{+}: U \rightarrow[0,1]$ and $\mu_{A}^{-}, \eta_{A}^{-}: U \rightarrow[-1,0]$ such that $0 \leq\left(\mu_{A}^{+}(x)\right)^{2}+\left(\eta_{A}^{+}(x)\right)^{2} \leq 1$ and $-1 \leq-\left[\left(\mu_{A}^{-}(x)\right)^{2}+\right.$ $\left.\left(\eta_{A}^{-}(x)\right)^{2}\right] \leq 0$. The degree of indeterminacy is determined as $\pi_{A}^{+}(x)=\left[\sqrt{1-\left(\mu_{A}^{+}(x)\right)^{2}-\left(\eta_{A}^{+}(x)\right)^{2}}\right]$ and $\pi_{A}^{-}(x)=-\left[\sqrt{1-\left(\mu_{A}^{-}(x)\right)^{2}-\left(\eta_{A}^{-}(x)\right)^{2}}\right]$. Here $A=\left\langle\mu_{A}^{+}, \eta_{A}^{+}, \mu_{A}^{-}, \eta_{A}^{-}\right\rangle$is called a Pythagorean bipolar fuzzy number (PBFN).

Proposition 1. [12] Let $\alpha_{1}=A\left(\mu_{\alpha_{1}}^{+}, \eta_{\alpha_{1}}^{+}, \mu_{\alpha_{1}}^{-}, \eta_{\alpha_{1}}^{-}\right), \alpha_{2}=A\left(\mu_{\alpha_{2}}^{+}, \eta_{\alpha_{2}}^{+}, \mu_{\alpha_{2}}^{-}, \eta_{\alpha_{2}}^{-}\right)$and $\alpha_{3}=A\left(\mu_{\alpha_{3}}^{+}, \eta_{\alpha_{3}}^{+}, \mu_{\alpha_{3}}^{-}, \eta_{\alpha_{3}}^{-}\right)$are any three PBFN's over $(U, E)$, then the following properties hold:

(i) $\alpha_{1}^{c}=\left(\eta_{\alpha_{1}}^{+}, \mu_{\alpha_{1}}^{+}, \eta_{\alpha_{1}}^{-}, \mu_{\alpha_{1}}^{-}\right)$;

(ii) $\alpha_{2} \uplus \alpha_{3}=\left[\max \left(\mu_{\alpha_{2}}^{+}, \mu_{\alpha_{3}}^{+}\right), \min \left(\eta_{\alpha_{2}}^{+}, \eta_{\alpha_{3}}^{+}\right), \min \left(\eta_{\alpha_{2},}^{-}, \eta_{\alpha_{3}}^{-}\right) \max \left(\mu_{\alpha_{2}}^{-}, \mu_{\alpha_{3}}^{-}\right)\right]$;

(iii) $\alpha_{2} \cap \alpha_{3}=\left[\min \left(\mu_{\alpha_{2}}^{+}, \mu_{\alpha_{3}}^{+}\right), \max \left(\eta_{\alpha_{2}}^{+}, \eta_{\alpha_{3}}^{+}\right), \max \left(\mu_{\alpha_{2}}^{-}, \mu_{\alpha_{3}}^{-}\right), \min \left(\eta_{\alpha_{2}}^{-}, \eta_{\alpha_{3}}^{-}\right)\right]$;

(iv) $\alpha_{2} \geq \alpha_{3}$ iff $\mu_{\alpha_{2}}^{+} \geq \mu_{\alpha_{3}}^{+}, \eta_{\alpha_{2}}^{+} \leq \eta_{\alpha_{3}}^{+}, \mu_{\alpha_{2}}^{-} \leq \mu_{\alpha_{3}}^{-}$and $\eta_{\alpha_{2}}^{-} \geq \eta_{\alpha_{3}}^{-}$;

(v) $\alpha_{2}=\alpha_{3}$ iff $\mu_{\alpha_{2}}^{+}=\mu_{\alpha_{3}}^{+}, \eta_{\alpha_{2}}^{+}=\eta_{\alpha_{3}}^{+}, \mu_{\alpha_{2}}^{-}=\mu_{\alpha_{3}}^{-}$and $\eta_{\alpha_{2}}^{-}=\eta_{\alpha_{3}}^{-}$.

Definition 3. [8] Let $U$ be a non-empty set of the universe and $E$ be a set of parameter. The pair $(\mathcal{F}, A)$ is called a bipolar fuzzy soft set (BFSS) on $U$ if $A \subseteq E$ and $\mathcal{F}: A \rightarrow \mathcal{B F}^{U}$, where $\mathcal{B F}^{U}$ is the set of all bipolar fuzzy subsets of $U$.

Definition 4. [13] Let $U$ be a non-empty set of the universe and $E$ be a set of parameter. The pair $(\mathcal{F}, A)$ is called a Pythagorean bipolar fuzzy soft set (PBFSS) on $U$ if $A \subseteq E$ and $\mathcal{F}: A \rightarrow P \mathcal{B} \mathcal{F}^{U}$, where $P \mathcal{B} \mathcal{F}^{U}$ is the set of all Pythagorean bipolar fuzzy subsets of $U$.

\section{Possibility Pythagorean bipolar fuzzy soft sets}

Definition 5. Let $U$ be a non-empty set of the universe, $E$ be a set of parameter and the pair $(U, E)$ is a soft universe. Further, let $\mathcal{F}: E \rightarrow \mathcal{B F}^{U}$ and $\mu$ be a bipolar fuzzy subset of $E$ such that $\mu: E \rightarrow \mathcal{B F}^{U}$. If $\mathcal{F}_{\mu}^{\mathcal{B}}: E \rightarrow$ $\mathcal{B F}^{U} \times \mathcal{B F}^{U}$ is a function defined as $\mathcal{F}_{\mu}^{\mathcal{B}}(e)=\{\langle\mathcal{B F}(e)(x), \mu(e)(x)\rangle, x \in U\}$ then $\mathcal{F}_{\mu}^{\mathcal{B}}$ is called a PBFSS over $(U, E)$.

Definition 6. Let $U$ be a non-empty set of the universe and $E$ be a set of parameter. The pair $(\mathcal{F}, A)$ is a PBFSS on $U$ if $\mathcal{F}: A \rightarrow P \mathcal{B} \mathcal{F}^{U}$, where $P \mathcal{B} \mathcal{F}^{U}$ is the set of all Pythagorean bipolar fuzzy subsets of $U$.

Example 1. A set of three Scooters $U=\left\{u_{1}, u_{2}, u_{3}\right\}$ under consideration and parameters $E=\left\{e_{1}=\right.$ Better Design, $e_{2}=$ Better Price, $e_{3}=$ More Mileage, $e_{4}=$ More Durable $\}$. Suppose $\mathcal{F}: E \rightarrow P \mathcal{B} \mathcal{F}^{U}$ is given by

$$
\begin{aligned}
& \mathcal{F}_{p}^{\mathcal{B}}\left(e_{1}\right)=\left\{\begin{array}{c}
\frac{u_{1}}{\langle 0.7,0.6,-0.3,-0.5\rangle} \\
\frac{u_{2}}{\langle 0.3,0.8,-0.8,-0.5\rangle} \\
\frac{u_{3}}{\langle 0.8,0.5,-0.4,-0.7\rangle}
\end{array}\right\} ; \\
& \mathcal{F}_{p}^{\mathcal{B}}\left(e_{2}\right)=\left\{\begin{array}{l}
\frac{u_{1}}{\langle 0.4,0.8,-0.7,-0.2\rangle} \\
\frac{u_{2}}{\langle 0.6,0.7,-0.8,-0.3\rangle} \\
\frac{u_{3}}{\langle 0.9,0.2,-0.7,-0.5\rangle}
\end{array}\right\} ; \\
& \mathcal{F}_{p}^{\mathcal{B}}\left(e_{3}\right)=\left\{\begin{array}{c}
\frac{u_{1}}{\langle 0.9,0.4,-0.2,-0.8\rangle} \\
\frac{u_{2}}{\langle 0.6,0.5,-0.4,-0.8\rangle} \\
\frac{u_{3}}{\langle 0.5,0.7,-0.9,-0.2\rangle}
\end{array}\right\} ;
\end{aligned}
$$




$$
\mathcal{F}_{p}^{\mathcal{B}}\left(e_{4}\right)=\left\{\begin{array}{c}
\frac{u_{1}}{\langle 0.7,0.6,-0.4,-0.7\rangle} \\
\frac{u_{2}}{\langle 0.8,0.5,-0.6,-0.8\rangle} \\
\frac{u_{3}}{\langle 0.6,0.8,-0.5,-0.6\rangle}
\end{array}\right\}
$$

The matrix form of $\mathcal{F}_{p}^{\mathcal{B}}$ can be written as:

$$
\left(\begin{array}{ccc}
\langle 0.7,0.6,-0.3,-0.5\rangle & \langle 0.3,0.8,-0.8,-0.5\rangle & \langle 0.8,0.5,-0.4,-0.7\rangle \\
\langle 0.4,0.8,-0.7,-0.2\rangle & \langle 0.6,0.7,-0.8,-0.3\rangle & \langle 0.9,0.2,-0.7,-0.5\rangle \\
\langle 0.9,0.4,-0.2,-0.8\rangle & \langle 0.6,0.5,-0.4,-0.8\rangle & \langle 0.5,0.7,-0.9,-0.2\rangle \\
\langle 0.7,0.6,-0.4,-0.7\rangle & \langle 0.8,0.5,-0.6,-0.8\rangle & \langle 0.6,0.8,-0.5,-0.6\rangle
\end{array}\right) .
$$

Definition 7. Let $U$ be a non-empty set of the universe, $E$ be a set of parameter and the pair ( $U, E)$ be a soft universe. Let $\mathcal{F}: E \rightarrow P \mathcal{B} \mathcal{F}^{U}$ and $\tilde{p}$ is a Pythagorean bipolar fuzzy subset of $E$. Further, let $p: E \rightarrow P \mathcal{B F}^{U}$ where $P \mathcal{B F}^{U}$ denotes the collection of all Pythagorean bipolar fuzzy subsets of $U$. If $\mathcal{F}_{p}^{\mathcal{B}}: E \rightarrow P \mathcal{B F} \mathcal{F}^{U} \times P \mathcal{B} \mathcal{F}^{U}$ is a function defined as $\mathcal{F}_{p}^{\mathcal{B}}(e)=\{\langle\mathcal{B F}(e)(x), p(e)(x)\rangle, x \in U\}$ then $\mathcal{F}_{p}^{\mathcal{B}}$ is a Possibility Pythagorean bipolar fuzzy soft sets (PPBFSS) on $(U, E)$ such that for each parameter $e, \mathcal{F}_{p}^{\mathcal{B}}(e)=$ $\left\{\left\langle x,\left(\mu_{\mathcal{F}(e)}^{+}(x), \eta_{\mathcal{F}(e)}^{+}(x), \mu_{\mathcal{F}(e)}^{-}(x), \eta_{\mathcal{F}(e)}^{-}(x)\right),\left(\mu_{p(e)}^{+}(x), \eta_{p(e)}^{+}(x), \mu_{p(e)}^{-}(x), \eta_{p(e)}^{-}(x)\right)\right\rangle, x \in U\right\}$.

Example 2. Let $U=\left\{u_{1}, u_{2}, u_{3}\right\}$ be a set of three cars under consideration and parameters $E=\left\{e_{1}=\right.$ Costly, $e_{2}=$ Attractive, $e_{3}=$ Better Fuel Efficient $\}$ is a set of parameters. Suppose that $\mathcal{F}_{p}^{\mathcal{B}}: E \rightarrow P \mathcal{B} \mathcal{F}^{U} \times P \mathcal{B} \mathcal{F}^{U}$ is given by

$$
\begin{aligned}
& \mathcal{F}_{p}^{\mathcal{B}}\left(e_{1}\right)=\left\{\begin{array}{c}
\frac{u_{1}}{\langle(0.6,0.7,-0.3,-0.8),(0.6,0.5,-0.8,-0.3)\rangle} \\
\frac{u_{2}}{\langle(0.9,0.4,-0.7,-0.5),(0.8,0.3,-0.6,-0.5)\rangle} \\
\frac{u_{3}}{\langle(0.8,0.5,-0.2,-0.9),(0.7,0.4,-0.8,-0.6)\rangle}
\end{array}\right\} ; \\
& \mathcal{F}_{p}^{\mathcal{B}}\left(e_{2}\right)=\left\{\begin{array}{l}
\frac{u_{1}}{\langle(0.7,0.4,-0.2,-0.8),(0.9,0.2,-0.7,-0.4)\rangle} \\
\frac{u_{2}}{\langle(0.3,0.9,-0.7,-0.4),(0.6,0.4,-0.6,-0.5)\rangle} \\
\frac{u_{3}}{\langle(0.5,0.6,-0.2,-0.9),(0.8,0.3,-0.7,-0.6)\rangle}
\end{array}\right\} ; \\
& \mathcal{F}_{p}^{\mathcal{B}}\left(e_{3}\right)=\left\{\begin{array}{c}
\frac{u_{1}}{\langle(0.3,0.7,-0.8,-0.4),(0.6,0.5,-0.7,-0.3)\rangle} \\
\frac{u_{2}}{\langle(0.8,0.4,-0.7,-0.3),(0.7,0.4,-0.6,-0.4)\rangle} \\
\frac{u_{3}}{\langle(0.9,0.2,-0.5,-0.6),(0.8,0.5,-0.9,-0.2)\rangle}
\end{array}\right\} .
\end{aligned}
$$

The matrix form of $\mathcal{F}_{p}^{\mathcal{B}}$ can be written as:

$$
\left(\begin{array}{llll}
\langle(0.6,0.7,-0.3,-0.8),(0.6,0.5,-0.8,-0.3)\rangle & \langle(0.9,0.4,-0.7,-0.5),(0.8,0.3,-0.6,-0.5)\rangle & \langle(0.8,0.5,-0.2,-0.9),(0.7,0.4,-0.8,-0.6)\rangle \\
\langle(0.7,0.4,-0.2,-0.8),(0.9,0.2,-0.7,-0.4)\rangle & \langle(0.3,0.9,-0.7,-0.4),(0.6,0.4,-0.6,-0.5)\rangle & \langle(0.5,0.6,-0.2,-0.9),(0.8,0.3,-0.7,-0.6)\rangle \\
\langle(0.3,0.7,-0.8,-0.4),(0.6,0.5,-0.7,-0.3)\rangle & \langle(0.8,0.4,-0.7,-0.3),(0.7,0.4,-0.6,-0.4)\rangle & \langle(0.9,0.2,-0.5,-0.6),(0.8,0.5,-0.9,-0.2)\rangle
\end{array}\right) .
$$

Definition 8. Let $U$ be a non-empty set of the universe and $E$ be a set of parameter. Suppose that $\mathcal{F}_{p}^{\mathcal{B}}$ and $\mathcal{G}_{q}^{\mathcal{B}}$ are two PPBFSSs on $(U, E)$. Now $\mathcal{F}_{p}^{\mathcal{B}} \sqsubseteq \mathcal{G}_{q}^{\mathcal{B}}$ if and only if

(i) $\mathcal{F}(e)(x) \sqsubseteq \mathcal{G}(e)(x)$ if

$$
\left\{\begin{array}{ll}
\mu_{\mathcal{F}(e)}^{+}(x) \leq \mu_{\mathcal{G}(e)}^{+}(x), & \eta_{\mathcal{F}(e)}^{+}(x) \geq \eta_{\mathcal{G}(e)}^{+}(x), \\
\mu_{\mathcal{F}(e)}^{-}(x) \geq \mu_{\mathcal{G}(e)}^{-}(x), \quad \eta_{\mathcal{F}(e)}^{-}(x) \leq \eta_{\mathcal{G}(e)}^{-}(x)
\end{array}\right\}
$$

(ii) $p(e)(x) \sqsubseteq q(e)(x)$ if

$$
\left\{\begin{array}{ll}
\mu_{p(e)}^{+}(x) \leq \mu_{q(e)}^{+}(x), & \eta_{p(e)}^{+}(x) \geq \eta_{q(e)}^{+}(x), \\
\mu_{p(e)}^{-}(x) \geq \mu_{q(e)}^{-}(x), & \eta_{p(e)}^{-}(x) \leq \eta_{q(e)}^{-}(x)
\end{array}\right\} ;
$$

$\forall e \in E$. 
Example 3. Consider the PPBFSS $\mathcal{F}_{p}^{\mathcal{B}}$ over $(U, E)$ as in Example 2. Let $\mathcal{G}_{q}^{\mathcal{B}}$ be another PPBFSS over $(U, E)$ defined as:

$$
\begin{aligned}
& \mathcal{G}_{q}^{\mathcal{B}}\left(e_{1}\right)=\left\{\begin{array}{l}
\frac{u_{1}}{\langle(0.7,0.5,-0.6,-0.7),(0.6,0.4,-0.9,-0.2)\rangle} \\
\frac{u_{2}}{\langle(0.9,0.2,-0.8,-0.4),(0.9,0.2,-0.7,-0.4)\rangle} \\
\frac{u_{3}}{\langle(0.9,0.1,-0.5,-0.8),(0.8,0.3,-0.9,-0.3)\rangle}
\end{array}\right\} ; \\
& \mathcal{G}_{q}^{\mathcal{B}}\left(e_{2}\right)=\left\{\begin{array}{c}
\frac{u_{1}}{\langle(0.8,0.3,-0.4,-0.6),(0.9,0.1,-0.8,-0.3)\rangle} \\
\langle(0.6,0.7,-0.8,-0.3),(0.7,0.4,-0.7,-0.4)\rangle \\
\frac{u_{3}}{\langle(0.7,0.4,-0.3,-0.7),(0.9,0.2,-0.8,-0.5)\rangle}
\end{array}\right\} ; \\
& \mathcal{G}_{q}^{\mathcal{B}}\left(e_{3}\right)=\left\{\begin{array}{c}
\frac{u_{1}}{\langle(0.5,0.6,-0.9,-0.3),(0.7,0.4,-0.9,-0.1)\rangle} \\
\left.\frac{u_{2}}{\langle(0.8,0.3,-0.8,-0.2),(0.8,0.3,-0.7,-0.3)\rangle}\right\rangle \\
\frac{u_{3}}{\langle(0.9,0.1,-0.7,-0.5),(0.9,0.4,-0.9,-0.2)\rangle}
\end{array}\right\}
\end{aligned}
$$

The matrix form of $\mathcal{G}_{q}^{\mathcal{B}}$ can be written as:

$$
\left(\begin{array}{llll}
\langle(0.7,0.5,-0.6,-0.7),(0.6,0.4,-0.9,-0.2)\rangle & \langle(0.9,0.2,-0.8,-0.4),(0.9,0.2,-0.7,-0.4)\rangle & \langle(0.9,0.1,-0.5,-0.8),(0.8,0.3,-0.9,-0.3)\rangle \\
\langle(0.8,0.3,-0.4,-0.6),(0.9,0.1,-0.8,-0.3)\rangle & \langle(0.6,0.7,-0.8,-0.3),(0.7,0.4,-0.7,-0.4)\rangle & \langle(0.7,0.4,-0.3,-0.7),(0.9,0.2,-0.8,-0.5)\rangle \\
\langle(0.5,0.6,-0.9,-0.3),(0.7,0.4,-0.9,-0.1)\rangle & \langle(0.8,0.3,-0.8,-0.2),(0.8,0.3,-0.7,-0.3)\rangle & \langle(0.9,0.1,-0.7,-0.5),(0.9,0.4,-0.9,-0.2)\rangle
\end{array}\right)
$$

Definition 9. Let $U$ be a non-empty set of the universe, $E$ be a set of parameter and $\mathcal{F}_{p}^{\mathcal{B}}$ be a PPBFSS on $(U, E)$. The complement of $\mathcal{F}_{p}^{\mathcal{B}}$ is denoted by $\mathcal{F}_{p^{c}}^{\mathcal{B}}$ and is defined by $\mathcal{F}_{p^{c}}^{\mathcal{B}}=\left\langle\mathcal{B F}^{c}(e)(x), p^{c}(e)(x)\right\rangle$, where $\mathcal{B F}^{c}(e)(x)=$ $\left(\eta_{\mathcal{F}(e)}^{+}(x), \mu_{\mathcal{F}(e)}^{+}(x), \eta_{\mathcal{F}(e)}^{-}(x), \mu_{\mathcal{F}(e)}^{-}(x)\right)$ and $p^{c}(e)(x)=\left(\eta_{p(e)}^{+}(x), \mu_{p(e)}^{+}(x), \eta_{p(e)}^{-}(x), \mu_{p(e)}^{-}(x)\right)$. It is true that $\mathcal{F}_{\left(p^{c}\right)^{c}}^{\mathcal{B}}=\mathcal{F}_{p}^{\mathcal{B}}$

Definition 10. Let $U$ be a non-empty set of the universe, $E$ be a set of parameter and $\mathcal{F}_{p}^{\mathcal{B}}$ and $\mathcal{G}_{q}^{\mathcal{B}}$ be two PPBFSSs on $(U, E)$. The union and intersection of $\mathcal{F}_{p}^{\mathcal{B}}$ and $\mathcal{G}_{q}^{\mathcal{B}}$ over $(U, E)$ are denoted by $\mathcal{F}_{p}^{\mathcal{B}} \cup \mathcal{G}_{q}^{\mathcal{B}}$ and $\mathcal{F}_{p}^{\mathcal{B}}$ ก $\mathcal{G}_{q}^{\mathcal{B}}$ respectively and are defined by $V_{v}: E \rightarrow P \mathcal{B F}^{U} \times P \mathcal{B F}^{U}, W_{w}: E \rightarrow P \mathcal{B} \mathcal{F}^{U} \times P \mathcal{B F}^{U}$ such that $V_{v}(e)(x)=$ $(V(e)(x), v(e)(x)), W_{w}(e)(x)=(W(e)(x), w(e)(x))$, where $V(e)(x)=\mathcal{F}(e)(x)$ U $\mathcal{G}(e)(x), v(e)(x)=p(e)(x)$ ש $q(e)(x), W(e)(x)=\mathcal{F}(e)(x) \cap \mathcal{G}(e)(x)$ and $w(e)(x)=p(e)(x) \cap q(e)(x)$, for all $x \in U$.

Example 4. Let $\mathcal{F}_{p}^{\mathcal{B}}$ and $\mathcal{G}_{q}^{\mathcal{B}}$ be the two PPBFSSs on $(U, E) . \mathcal{F}_{p}^{\mathcal{B}}$ is same as in Example 2 and $\mathcal{G}_{q}^{\mathcal{B}}$ is defined as,

$$
\begin{aligned}
& \mathcal{G}_{q}^{\mathcal{B}}\left(e_{1}\right)=\left\{\begin{array}{l}
\left.\frac{u_{1}}{\langle(0.3,0.4,-0.2,-0.3),(0.5,0.4,-0.3,-0.1)\rangle}\right\rangle \\
\frac{u_{2}}{\langle(0.4,0.5,-0.6,-0.2),(0.6,0.2,-0.4,-0.2)\rangle} \\
\left.\frac{u_{3}}{\langle(0.6,0.2,-0.1,-0.4),(0.4,0.3,-0.5,-0.6)\rangle}\right\rangle
\end{array} ;\right. \\
& \mathcal{G}_{q}^{\mathcal{B}}\left(e_{2}\right)=\left\{\begin{array}{l}
\frac{u_{1}}{\langle(0.8,0.7,-0.4,-0.3),(0.2,0.1,-0.3,-0.5)\rangle} \\
\frac{u_{2}}{\langle(0.6,0.4,-0.3,-0.8),(0.3,0.4,-0.2,-0.8)\rangle} \\
\left.\frac{u_{3}}{\langle(0.5,0.3,-0.5,-0.4),(0.4,0.3,-0.6,-0.9)\rangle}\right\rangle
\end{array}\right\} ; \\
& \mathcal{G}_{q}^{\mathcal{B}}\left(e_{3}\right)=\left\{\begin{array}{l}
\frac{u_{1}}{\langle(0.6,0.4,-0.4,-0.1),(0.5,0.6,-0.3,-0.4)\rangle} \\
\frac{u_{2}}{\langle(0.7,0.9,-0.6,-0.4),(0.6,0.1,-0.8,-0.5)\rangle}, \\
\frac{u_{3}}{\langle(0.2,0.6,-0.3,-0.2),(0.3,0.2,-0.7,-0.1)\rangle}
\end{array}\right\} .
\end{aligned}
$$

The matrix form of $\mathcal{F}_{p}^{\mathcal{B}} \cup \mathcal{G}_{q}^{\mathcal{B}}$ can be written as:

$$
\left(\begin{array}{llll}
\langle(0.6,0.4,-0.3,-0.3),(0.6,0.4,-0.8,-0.1)\rangle & \langle(0.9,0.4,-0.7,-0.2),(0.8,0.2,-0.6,-0.2)\rangle & \langle(0.8,0.2,-0.2,-0.4),(0.7,0.3,-0.8,-0.6)\rangle \\
\langle(0.8,0.4,-0.4,-0.3),(0.9,0.1,-0.7,-0.4)\rangle & \langle(0.6,0.4,-0.4,-0.4),(0.6,0.4,-0.6,-0.5)\rangle & \langle(0.5,0.3,-0.5,-0.4),(0.8,0.3,-0.7,-0.6)\rangle \\
\langle(0.6,0.4,-0.8,-0.1),(0.6,0.5,-0.7,-0.3)\rangle & \langle(0.8,0.4,-0.7,-0.3),(0.7,0.1,-0.8,-0.4)\rangle & \langle(0.9,0.2,-0.5,-0.2),(0.8,0.2,-0.9,-0.1)\rangle
\end{array}\right)
$$


and the matrix form of $\mathcal{F}_{p}^{\mathcal{B}} \cap \mathcal{G}_{q}^{\mathcal{B}}$ can be written as:

$\left(\begin{array}{llll}\langle(0.3,0.7,-0.2,-0.8),(0.5,0.5,-0.3,-0.3)\rangle & \langle(0.4,0.5,-0.6,-0.5),(0.6,0.3,-0.4,-0.5)\rangle & \langle(0.6,0.5,-0.1,-0.9),(0.4,0.4,-0.5,-0.6)\rangle \\ \langle(0.7,0.7,-0.2,-0.8),(0.2,0.2,-0.3,-0.5)\rangle & \langle(0.3,0.9,-0.3,-0.8),(0.3,0.4,-0.2,-0.8)\rangle & \langle(0.5,0.6,-0.2,-0.9),(0.4,0.3,-0.6,-0.9)\rangle \\ \langle(0.3,0.7,-0.4,-0.4),(0.5,0.6,-0.3,-0.4)\rangle & \langle(0.7,0.9,-0.6,-0.4),(0.6,0.4,-0.6,-0.5)\rangle & \langle(0.2,0.6,-0.3,-0.6),(0.3,0.5,-0.7,-0.2)\rangle\end{array}\right)$.

Definition 11. A PPBFSS $\Theta_{\theta}^{\mathcal{B}}(e)(x)=\langle\Theta(e)(x), \theta(e)(x)\rangle$ is said to a possibility null Pythagorean bipolar fuzzy soft set $\Theta_{\theta}^{\mathcal{B}}: E \rightarrow P \mathcal{B F}^{U} \times P \mathcal{B F} \mathcal{F}^{U}$, where $\Theta^{+}(e)(x)=(0,1), \theta^{+}(e)(x)=(0,1), \Theta^{-}(e)(x)=(-1,0)$ and $\theta^{-}(e)(x)=$ $(-1,0), \forall x \in U$.

Definition 12. A PPBFSS $\Omega_{\omega}^{\mathcal{B}}(e)(x)=\langle\Omega(e)(x), \omega(e)(x)\rangle$ is said to a possibility absolute Pythagorean bipolar fuzzy soft set $\Omega_{\omega}^{\mathcal{B}}: E \rightarrow P \mathcal{B} \mathcal{F}^{U} \times P \mathcal{B} \mathcal{F}^{U}$, where $\Omega^{+}(e)(x)=(1,0), \omega^{+}(e)(x)=(1,0), \Omega^{-}(e)(x)=(0,-1)$ and $\omega^{-}(e)(x)=(0,-1), \forall x \in U$.

Theorem 1. Let $\mathcal{F}_{p}^{\mathcal{B}}$ be a PPBFSS on $(U, E)$. Then the following properties hold:

(i) $\mathcal{F}_{p}^{\mathcal{B}}=\mathcal{F}_{p}^{\mathcal{B}} \uplus \mathcal{F}_{p}^{\mathcal{B}}, \mathcal{F}_{p}^{\mathcal{B}}=\mathcal{F}_{p}^{\mathcal{B}} \cap \mathcal{F}_{p}^{\mathcal{B}}$;

(ii) $\mathcal{F}_{p}^{\mathcal{B}} \sqsubseteq \mathcal{F}_{p}^{\mathcal{B}} \amalg \mathcal{F}_{p}^{\mathcal{B}}, \mathcal{F}_{p}^{\mathcal{B}} \sqsubseteq \mathcal{F}_{p}^{\mathcal{B}}$ ก $\mathcal{F}_{p}^{\mathcal{B}}$;

(iii) $\mathcal{F}_{p}^{\mathcal{B}} \uplus \Theta_{\theta}^{\mathcal{B}}=\mathcal{F}_{p}^{\mathcal{B}}, \mathcal{F}_{p}^{\mathcal{B}} \cap \Theta_{\theta}^{\mathcal{B}}=\Theta_{\theta}^{\mathcal{B}}$;

(iv) $\mathcal{F}_{p}^{\mathcal{B}} \uplus \Omega_{\omega}^{\mathcal{B}}=\Omega_{\omega}^{\mathcal{B}}, \mathcal{F}_{p}^{\mathcal{B}} \cap \Omega_{\omega}^{\mathcal{B}}=\mathcal{F}_{p}^{\mathcal{B}}$.

Remark 1. Let $\mathcal{F}_{p}^{\mathcal{B}}$ be a PPBFSS on $(U, E)$. If $\mathcal{F}_{p}^{\mathcal{B}} \neq \Omega_{\omega}^{\mathcal{B}}$ or $\mathcal{F}_{p}^{\mathcal{B}} \neq \Theta_{\theta}^{\mathcal{B}}$ then $\mathcal{F}_{p}^{\mathcal{B}} \uplus \mathcal{F}_{p^{c}}^{\mathcal{B}} \neq \Omega_{\omega}^{\mathcal{B}}$ and $\mathcal{F}_{p}^{\mathcal{B}}$ ก $\mathcal{F}_{p^{c}}^{\mathcal{B}} \neq \Theta_{\theta}^{\mathcal{B}}$.

Theorem 2. Let $\mathcal{F}_{p}^{\mathcal{B}}, \mathcal{G}_{q}^{\mathcal{B}}$ and $\mathcal{H}_{r}^{\mathcal{B}}$ are three PPBFSSs over $(U, E)$. Then the following properties hold:

(1) $\mathcal{F}_{p}^{\mathcal{B}} \cup \mathcal{G}_{q}^{\mathcal{B}}=\mathcal{G}_{q}^{\mathcal{B}} \cup \mathcal{F}_{p}^{\mathcal{B}}$;

(2) $\mathcal{F}_{p}^{\mathcal{B}} \cap \mathcal{G}_{q}^{\mathcal{B}}=\mathcal{G}_{q}^{\mathcal{B}} \cap \mathcal{F}_{p}^{\mathcal{B}}$;

(3) $\mathcal{F}_{p}^{\mathcal{B}} \amalg\left(\mathcal{G}_{q}^{\mathcal{B}} \uplus \mathcal{H}_{r}^{\mathcal{B}}\right)=\left(\mathcal{F}_{p}^{\mathcal{B}} \cup \mathcal{G}_{q}^{\mathcal{B}}\right) \cup \mathcal{H}_{r}^{\mathcal{B}}$;

(4) $\mathcal{F}_{p}^{\mathcal{B}} \cap\left(\mathcal{G}_{q}^{\mathcal{B}} \cap \mathcal{H}_{r}^{\mathcal{B}}\right)=\left(\mathcal{F}_{p}^{\mathcal{B}} \cap \mathcal{G}_{q}^{\mathcal{B}}\right) \cap \mathcal{H}_{r}^{\mathcal{B}}$;

(5) $\left(\mathcal{F}_{p}^{\mathcal{B}} \uplus \mathcal{G}_{q}^{\mathcal{B}}\right)^{c}=\mathcal{F}_{p^{c}}^{\mathcal{B}} \cap \mathcal{G}_{q^{c}}^{\mathcal{B}}$;

(6) $\left(\mathcal{F}_{p}^{\mathcal{B}} \cap \mathcal{G}_{q}^{\mathcal{B}}\right)^{c}=\mathcal{F}_{p^{c}}^{\mathcal{B}} \cup \mathcal{G}_{q^{c}}^{\mathcal{B}}$;

(7) $\left(\mathcal{F}_{p}^{\mathcal{B}} \uplus \mathcal{G}_{q}^{\mathcal{B}}\right) \cap \mathcal{F}_{p}^{\mathcal{B}}=\mathcal{F}_{p}^{\mathcal{B}}$;

(8) $\left(\mathcal{F}_{p}^{\mathcal{B}} \cap \mathcal{G}_{q}^{\mathcal{B}}\right) \cup \mathcal{F}_{p}^{\mathcal{B}}=\mathcal{F}_{p}^{\mathcal{B}}$;

(9) $\mathcal{F}_{p}^{\mathcal{B}} \uplus\left(\mathcal{G}_{q}^{\mathcal{B}}\right.$ ก $\left.\mathcal{H}_{r}^{\mathcal{B}}\right)=\left(\mathcal{F}_{p}^{\mathcal{B}} \uplus \mathcal{G}_{q}^{\mathcal{B}}\right) \cap\left(\mathcal{F}_{p}^{\mathcal{B}} \uplus \mathcal{H}_{r}^{\mathcal{B}}\right)$;

(10) $\mathcal{F}_{p}^{\mathcal{B}}$ ก $\left(\mathcal{G}_{q}^{\mathcal{B}} \uplus \mathcal{H}_{r}^{\mathcal{B}}\right)=\left(\mathcal{F}_{p}^{\mathcal{B}} \cap \mathcal{G}_{q}^{\mathcal{B}}\right) \cup\left(\mathcal{F}_{p}^{\mathcal{B}} \cap \mathcal{H}_{r}^{\mathcal{B}}\right)$.

Proof. The proof follows from Definition 9 and Definition 10.

Definition 13. Let $\left(\mathcal{F}_{p}^{\mathcal{B}}, A\right)$ and $\left(\mathcal{G}_{q}^{\mathcal{B}}, B\right)$ be two PPBFSSs on $(U, E)$, then the operations " $\left(\mathcal{F}_{p}^{\mathcal{B}}, A\right) \operatorname{AND}\left(\mathcal{G}_{q}^{\mathcal{B}}, B\right)$ " is denoted by $\left(\mathcal{F}_{p}^{\mathcal{B}}, A\right) \wedge\left(\mathcal{G}_{q}^{\mathcal{B}}, B\right)$ and is defined by $\left(\mathcal{F}_{p}^{\mathcal{B}}, A\right) \wedge\left(\mathcal{G}_{q}^{\mathcal{B}}, B\right)=\left(\mathcal{H}_{r}^{\mathcal{B}}, A \times B\right)$, where $\mathcal{H}_{r}^{\mathcal{B}}(\kappa, \lambda)=(\mathcal{H}(\kappa, \lambda)(x), r(\kappa, \lambda)(x))$ such that $\mathcal{H}(\kappa, \lambda)=\mathcal{F}(\kappa) \cap \mathcal{G}(\lambda)$ and $r(\kappa, \lambda)=p(\kappa)$ ก $q(\lambda)$, for all $(\kappa, \lambda) \in A \times B$.

Definition 14. Let $\left(\mathcal{F}_{p}^{\mathcal{B}}, A\right)$ and $\left(\mathcal{G}_{q}^{\mathcal{B}}, B\right)$ be two PPBFSSs on $(U, E)$, then the operations " $\left(\mathcal{F}_{p}^{\mathcal{B}}, A\right)$ OR $\left(\mathcal{G}_{q}^{\mathcal{B}}, B\right)^{\prime \prime}$ is denoted by $\left(\mathcal{F}_{p}^{\mathcal{B}}, A\right) \vee\left(\mathcal{G}_{q}^{\mathcal{B}}, B\right)$ and is defined by $\left(\mathcal{F}_{p}^{\mathcal{B}}, A\right) \vee\left(\mathcal{G}_{q}^{\mathcal{B}}, B\right)=\left(\mathcal{H}_{r}^{\mathcal{B}}, A \times B\right)$, where $\mathcal{H}_{r}^{\mathcal{B}}(\kappa, \lambda)=$ $(\mathcal{H}(\kappa, \lambda)(x), r(\kappa, \lambda)(x))$ such that $\mathcal{H}(\kappa, \lambda)=\mathcal{F}(\kappa) \cup \mathcal{G}(\lambda)$ and $r(\kappa, \lambda)=p(\kappa) \cup q(\lambda)$, for all $(\kappa, \lambda) \in A \times B$.

Theorem 3. Let $\left(\mathcal{F}_{p}^{\mathcal{B}}, A\right)$ and $\left(\mathcal{G}_{q}^{\mathcal{B}}, B\right)$ be two PPBFSSs on $(U, E)$, then

(i) $\left(\left(\mathcal{F}_{p}^{\mathcal{B}}, A\right) \wedge\left(\mathcal{G}_{q}^{\mathcal{B}}, B\right)\right)^{\mathcal{c}}=\left(\mathcal{F}_{p^{c}}^{\mathcal{B}}, A\right) \vee\left(\mathcal{G}_{q^{c}}^{\mathcal{B}}, B\right)$;

(ii) $\left(\left(\mathcal{F}_{p}^{\mathcal{B}}, A\right) \vee\left(\mathcal{G}_{q}^{\mathcal{B}}, B\right)\right)^{\mathcal{C}}=\left(\mathcal{F}_{p^{c}}^{\mathcal{B}}, A\right) \wedge\left(\mathcal{G}_{q^{c}}^{\mathcal{B}}, B\right)$. 
Proof. (i) Suppose that $\left(\mathcal{F}_{p}^{\mathcal{B}}, A\right) \wedge\left(\mathcal{G}_{q}^{\mathcal{B}}, B\right)=\left(\mathcal{H}_{r}^{\mathcal{B}}, A \times B\right)$. Now, $\mathcal{H}_{r^{c}}^{\mathcal{B}}(\kappa, \lambda)=\left(\mathcal{H}^{c}(\kappa, \lambda)(x), r^{c}(\kappa, \lambda)(x)\right)$, for all $(\kappa, \lambda) \in A \times B$. By Theorem 2 and Definition 13, $\mathcal{H}^{c}(\kappa, \lambda)=(\mathcal{F}(\kappa) \cap \mathcal{G}(\lambda))^{c}=\mathcal{F}^{c}(\kappa) \cup \mathcal{G}^{c}(\lambda)$ and $r^{c}(\kappa, \lambda)=(p(\kappa) \cap q(\lambda))^{c}=p^{c}(\kappa) \cup q^{c}(\lambda)$.

On the other hand, given that $\left(\mathcal{F}_{p^{c}}^{\mathcal{B}}, A\right) \vee\left(\mathcal{G}_{q^{c}}^{\mathcal{B}}, B\right)=\left(\omega_{0}, A \times B\right)$, where $\omega_{0}(\kappa, \lambda)=(\omega(\kappa, \lambda)(x), o(\kappa, \lambda)(x))$ such that $\omega(\kappa, \lambda)=\mathcal{F}^{c}(\kappa) \cup \mathcal{G}^{c}(\lambda)$ and $o(\kappa, \lambda)=p^{c}(\kappa) \cup q^{c}(\lambda)$ for all $(\kappa, \lambda) \in A \times B$. Thus, $\mathcal{H}_{r}^{c}=\omega_{0}$. Hence $\left(\left(\mathcal{F}_{p}^{\mathcal{B}}, A\right) \wedge\left(\mathcal{G}_{q}^{\mathcal{B}}, B\right)\right)^{c}=\left(\mathcal{F}_{p^{c}}^{\mathcal{B}}, A\right) \vee\left(\mathcal{G}_{q^{c}}^{\mathcal{B}}, B\right)$.

(ii) The proof is similarly to (i).

\section{Similarity measure between two PPBFSSs}

Definition 15. Let $U$ be a non-empty set of the universe, $E$ be a set of parameter and $\mathcal{F}_{p}^{\mathcal{B}}$ and $\mathcal{G}_{q}^{\mathcal{B}}$ be two PPBFSSs on $(U, E)$. The similarity measure between two PPBFSSs $\mathcal{F}_{p}^{\mathcal{B}}$ and $\mathcal{G}_{q}^{\mathcal{B}}$ is denoted by $\operatorname{Sim}\left(\mathcal{F}_{p}^{\mathcal{B}}, \mathcal{G}_{q}^{\mathcal{B}}\right)$ and is defined as $\operatorname{Sim}\left(\mathcal{F}_{p}^{\mathcal{B}}, \mathcal{G}_{q}^{\mathcal{B}}\right)=\left[\Phi^{\mathcal{B}}(\mathcal{F}, \mathcal{G}) \cdot \Psi^{\mathcal{B}}(p, q)\right]$ such that

$$
\Phi^{\mathcal{B}}(\mathcal{F}, \mathcal{G})=\frac{\mathbb{T}^{\mathcal{B}}(\mathcal{F}(e)(x), \mathcal{G}(e)(x))+\mathbb{S}^{\mathcal{B}}(\mathcal{F}(e)(x), \mathcal{G}(e)(x))}{2} \text { and } \Psi^{\mathcal{B}}(p, q)=1-\frac{\sum\left|\alpha_{i}-\beta_{i}\right|}{\sum\left|\alpha_{i}+\beta_{i}\right|}
$$

where

and

$$
\begin{aligned}
& \left.\left.\mathbb{T}^{\mathcal{B}}(\mathcal{F}(e)(x), \mathcal{G}(e)(x))=\frac{\sum_{i=1}^{n}\left[\left[\mu_{\mathcal{F}\left(e_{i}\right)}^{+}(x) \cdot \mu_{\mathcal{G}\left(e_{i}\right)}^{+}(x)\right]+\left[\mu_{\mathcal{F}\left(e_{i}\right)}^{-}(x) \cdot \mu_{\mathcal{G}\left(e_{i}\right)}^{-}(x)\right]\right]}{\sum_{i=1}^{n}\left[\left[1-\sqrt{\left[\left(1-\mu_{\mathcal{F}\left(e_{i}\right)}^{2+}(x)\right) \cdot\left(1-\mu_{\mathcal{G}\left(e_{i}\right)}^{2+}(x)\right)\right]}\right]+\left[1-\sqrt{\left[\left(1-\mu_{\mathcal{F}\left(e_{i}\right)}^{2-}(x)\right) \cdot\left(1-\mu_{\mathcal{G}\left(e_{i}\right)}^{2-}(x)\right)\right.}\right]\right.}\right]\right]^{\prime} \\
& \mathbb{S}^{\mathcal{B}}(\mathcal{F}(e)(x), \mathcal{G}(e)(x))=\sqrt{1-\frac{\sum_{i=1}^{n}\left[\left|\eta_{\mathcal{F}\left(e_{i}\right)}^{2+}(x)-\eta_{\mathcal{G}\left(e_{i}\right)}^{2+}(x)\right|+\left|\eta_{\mathcal{F}\left(e_{i}\right)}^{2-}(x)-\eta_{\mathcal{G}\left(e_{i}\right)}^{2-}(x)\right|\right]}{\sum_{i=1}^{n}\left[\left[1+\left[\left(\eta_{\mathcal{F}\left(e_{i}\right)}^{2+}(x)\right) \cdot\left(\eta_{\mathcal{G}\left(e_{i}\right)}^{2+}(x)\right)\right]\right]+\left[1+\left[\left(\eta_{\mathcal{F}\left(e_{i}\right)}^{2-}(x)\right) \cdot\left(\eta_{\mathcal{G}\left(e_{i}\right)}^{2-}(x)\right)\right]\right]\right.}}, \\
& \alpha_{i}=\frac{\mu_{p\left(e_{i}\right)}^{2+}(x)+\mu_{p\left(e_{i}\right)}^{2-}(x)}{\left[\mu_{p\left(e_{i}\right)}^{2+}(x)+\eta_{p\left(e_{i}\right)}^{2+}(x)\right]+\left[\mu_{p\left(e_{i}\right)}^{2-}(x)+\eta_{p\left(e_{i}\right)}^{2-}(x)\right]}
\end{aligned}
$$

$$
\beta_{i}=\frac{\mu_{q\left(e_{i}\right)}^{2+}(x)+\mu_{q\left(e_{i}\right)}^{2-}(x)}{\left[\mu_{q\left(e_{i}\right)}^{2+}(x)+\eta_{q\left(e_{i}\right)}^{2+}(x)\right]+\left[\mu_{q\left(e_{i}\right)}^{2-}(x)+\eta_{q\left(e_{i}\right)}^{2-}(x)\right]} .
$$

Theorem 4. Let $\mathcal{F}_{p}^{\mathcal{B}}, \mathcal{G}_{q}^{\mathcal{B}}$ and $\mathcal{H}_{r}^{\mathcal{B}}$ be the any three PPBFSSs over $(U, E)$. Then the following statements hold:

(i) $\operatorname{Sim}\left(\mathcal{F}_{p}^{\mathcal{B}}, \mathcal{G}_{q}^{\mathcal{B}}\right)=\operatorname{Sim}\left(\mathcal{G}_{q}^{\mathcal{B}}, \mathcal{F}_{p}^{\mathcal{B}}\right)$;

(ii) $0 \leq \operatorname{Sim}\left(\mathcal{F}_{p}^{\mathcal{B}}, \mathcal{G}_{q}^{\mathcal{B}}\right) \leq 1$;

(iii) $\mathcal{F}_{p}^{\mathcal{B}}=\mathcal{G}_{q}^{\mathcal{B}} \Longrightarrow \operatorname{Sim}\left(\mathcal{F}_{p}^{\mathcal{B}}, \mathcal{G}_{q}^{\mathcal{B}}\right)=1$;

(iv) $\mathcal{F}_{p}^{\mathcal{B}} \sqsubseteq \mathcal{G}_{q}^{\mathcal{B}} \sqsubseteq \mathcal{H}_{r}^{\mathcal{B}} \Longrightarrow \operatorname{Sim}\left(\mathcal{F}_{p}^{\mathcal{B}}, \mathcal{H}_{r}^{\mathcal{B}}\right) \leq \operatorname{Sim}\left(\mathcal{G}_{q}^{\mathcal{B}}, \mathcal{H}_{r}^{\mathcal{B}}\right)$;

(v) $\mathcal{F}_{p}^{\mathcal{B}}$ ก $\mathcal{G}_{q}^{\mathcal{B}}=\{\phi\} \Leftrightarrow \operatorname{Sim}\left(\mathcal{F}_{p}^{\mathcal{B}}, \mathcal{G}_{q}^{\mathcal{B}}\right)=0$.

Proof. The proofs of (i), (ii) and (v) are trivial.

(iii) Given that $\mathcal{F}_{p}^{\mathcal{B}}=\mathcal{G}_{q}^{\mathcal{B}}$. Now,

$$
\begin{aligned}
\mathbb{T}^{\mathcal{B}}(\mathcal{F}(e)(x), \mathcal{G}(e)(x)) & =\frac{\sum_{i=1}^{n}\left[\mu_{\mathcal{F}\left(e_{i}\right)}^{2+}(x)+\mu_{\mathcal{F}\left(e_{i}\right)}^{2-}(x)\right]}{\sum_{i=1}^{n}\left[\left[1-1+\mu_{\mathcal{F}\left(e_{i}\right)}^{2+}(x)\right]+\left[1-1+\mu_{\mathcal{F}\left(e_{i}\right)}^{2-}(x)\right]\right]} \\
& =\frac{\sum_{i=1}^{n}\left[\mu_{\mathcal{F}\left(e_{i}\right)}^{2+}(x)+\mu_{\mathcal{F}\left(e_{i}\right)}^{2-}(x)\right]}{\sum_{i=1}^{n}\left[\mu_{\mathcal{F}\left(e_{i}\right)}^{2+}(x)+\mu_{\mathcal{F}\left(e_{i}\right)}^{2-}(x)\right]} \\
& =1,
\end{aligned}
$$

and

$$
\mathbb{S}^{\mathcal{B}}(\mathcal{F}(e)(x), \mathcal{G}(e)(x))=\sqrt{(1-0)}=1 .
$$


Thus, $\Phi^{\mathcal{B}}(\mathcal{F}, \mathcal{G})=\frac{1+1}{2}=1$ and $\Psi^{\mathcal{B}}(p, q)=1$. Hence $\operatorname{Sim}\left(\mathcal{F}_{p}^{\mathcal{B}}, \mathcal{G}_{q}^{\mathcal{B}}\right)=1$. This proves (iii).

(iv) Given that

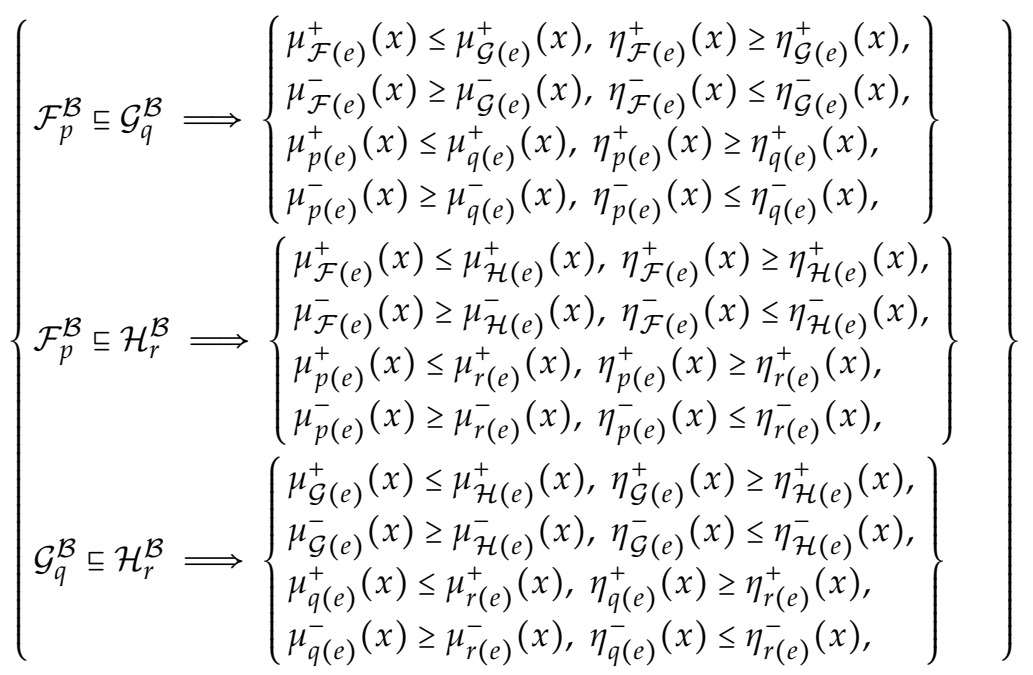

Clearly,

$$
\mu_{\mathcal{F}(e)}^{+}(x) \cdot \mu_{\mathcal{H}(e)}^{+}(x) \leq \mu_{\mathcal{G}(e)}^{+}(x) \cdot \mu_{\mathcal{H}(e)}^{+}(x)
$$

and

$$
\mu_{\mathcal{F}(e)}^{-}(x) \cdot \mu_{\mathcal{H}(e)}^{-}(x) \leq \mu_{\mathcal{G}(e)}^{-}(x) \cdot \mu_{\mathcal{H}(e)}^{-}(x)
$$

implies that

$\sum_{i=1}^{n}\left[\left[\mu_{\mathcal{F}\left(e_{i}\right)}^{+}(x) \cdot \mu_{\mathcal{H}\left(e_{i}\right)}^{+}(x)\right]+\left[\mu_{\mathcal{F}\left(e_{i}\right)}^{-}(x) \cdot \mu_{\mathcal{H}\left(e_{i}\right)}^{-}(x)\right]\right] \leq \sum_{i=1}^{n}\left[\left[\mu_{\mathcal{G}\left(e_{i}\right)}^{+}(x) \cdot \mu_{\mathcal{H}\left(e_{i}\right)}^{+}(x)\right]+\left[\mu_{\mathcal{G}\left(e_{i}\right)}^{-}(x) \cdot \mu_{\mathcal{H}\left(e_{i}\right)}^{-}(x)\right]\right]$.

Also, clearly

$$
\left(\mu_{\mathcal{F}(e)}^{+}(x)\right)^{2} \leq\left(\mu_{\mathcal{G}(e)}^{+}(x)\right)^{2}
$$

and

$$
\left(\mu_{\mathcal{F}(e)}^{-}(x)\right)^{2} \leq\left(\mu_{\mathcal{G}(e)}^{-}(x)\right)^{2}
$$

implies that

$$
\left[\left(1-\left(\mu_{\mathcal{F}(e)}^{+}(x)\right)^{2}\right) \cdot\left(1-\left(\mu_{\mathcal{H}(e)}^{+}(x)\right)^{2}\right)\right] \geq\left[\left(1-\left(\mu_{\mathcal{G}(e)}^{+}(x)\right)^{2}\right) \cdot\left(1-\left(\mu_{\mathcal{H}(e)}^{+}(x)\right)^{2}\right)\right]
$$

and

$$
\left[1-\sqrt{\left[\left(1-\left(\mu_{\mathcal{F}(e)}^{+}(x)\right)^{2}\right) \cdot\left(1-\left(\mu_{\mathcal{H}(e)}^{+}(x)\right)^{2}\right)\right]}\right] \leq\left[1-\sqrt{\left[\left(1-\left(\mu_{\mathcal{G}(e)}^{+}(x)\right)^{2}\right) \cdot\left(1-\left(\mu_{\mathcal{H}(e)}^{+}(x)\right)^{2}\right)\right]}\right] .
$$

Similarly,

$$
\left[1-\sqrt{\left[\left(1-\left(\mu_{\mathcal{F}(e)}^{-}(x)\right)^{2}\right) \cdot\left(1-\left(\mu_{\mathcal{H}(e)}^{-}(x)\right)^{2}\right)\right]}\right] \leq\left[1-\sqrt{\left[\left(1-\left(\mu_{\mathcal{G}(e)}^{-}(x)\right)^{2}\right) \cdot\left(1-\left(\mu_{\mathcal{H}(e)}^{-}(x)\right)^{2}\right)\right]}\right] .
$$

By adding (2) and (3), we get

$$
\begin{aligned}
& {\left[1-\sqrt{\left[\left(1-\left(\mu_{\mathcal{F}(e)}^{+}(x)\right)^{2}\right) \cdot\left(1-\left(\mu_{\mathcal{H}(e)}^{+}(x)\right)^{2}\right)\right]}\right]+\left[1-\sqrt{\left[\left(1-\left(\mu_{\mathcal{F}(e)}^{-}(x)\right)^{2}\right) \cdot\left(1-\left(\mu_{\mathcal{H}(e)}^{-}(x)\right)^{2}\right)\right]}\right]} \\
& \leq\left[1-\sqrt{\left[\left(1-\left(\mu_{\mathcal{G}(e)}^{+}(x)\right)^{2}\right) \cdot\left(1-\left(\mu_{\mathcal{H}(e)}^{+}(x)\right)^{2}\right)\right]}\right]+\left[1-\sqrt{\left[\left(1-\left(\mu_{\mathcal{G}(e)}^{-}(x)\right)^{2}\right) \cdot\left(1-\left(\mu_{\mathcal{H}(e)}^{-}(x)\right)^{2}\right)\right]}\right] .
\end{aligned}
$$


Open J. Discret. Appl. Math. 2021, 4(2), 17-29

24

Hence,

$$
\begin{aligned}
& \sum_{i=1}^{n}\left[\left[1-\sqrt{\left[\left(1-\left(\mu_{\mathcal{F}(e)}^{+}(x)\right)^{2}\right) \cdot\left(1-\left(\mu_{\mathcal{H}(e)}^{+}(x)\right)^{2}\right)\right]}\right]+\left[1-\sqrt{\left[\left(1-\left(\mu_{\mathcal{F}(e)}^{-}(x)\right)^{2}\right) \cdot\left(1-\left(\mu_{\mathcal{H}(e)}^{-}(x)\right)^{2}\right)\right]}\right]\right] \\
& \left.\leq \sum_{i=1}^{n}\left[\left[1-\sqrt{\left[\left(1-\left(\mu_{\mathcal{G}(e)}^{+}(x)\right)^{2}\right) \cdot\left(1-\left(\mu_{\mathcal{H}(e)}^{+}(x)\right)^{2}\right)\right.}\right]\right]+\left[1-\sqrt{\left[\left(1-\left(\mu_{\mathcal{G}(e)}^{-}(x)\right)^{2}\right) \cdot\left(1-\left(\mu_{\mathcal{H}(e)}^{-}(x)\right)^{2}\right)\right]}\right]\right]
\end{aligned}
$$

Dividing (1) by (4), we get

$$
\begin{gathered}
\frac{\sum_{i=1}^{n}\left[\left[\mu_{\mathcal{F}\left(e_{i}\right)}^{+}(x) \cdot \mu_{\mathcal{H}\left(e_{i}\right)}^{+}(x)\right]+\left[\mu_{\mathcal{F}\left(e_{i}\right)}^{-}(x) \cdot \mu_{\mathcal{H}\left(e_{i}\right)}^{-}(x)\right]\right]}{\sum_{i=1}^{n}\left[\left[1-\sqrt{\left[\left(1-\mu_{\mathcal{F}\left(e_{i}\right)}^{2+}(x)\right) \cdot\left(1-\mu_{\mathcal{H}\left(e_{i}\right)}^{2+}(x)\right)\right]}\right]+\left[1-\sqrt{\left[\left(1-\mu_{\mathcal{F}\left(e_{i}\right)}^{2-}(x)\right) \cdot\left(1-\mu_{\mathcal{H}\left(e_{i}\right)}^{2-}(x)\right)\right]}\right]\right.} \\
\leq \frac{\sum_{i=1}^{n}\left[\left[\mu_{\mathcal{G}\left(e_{i}\right)}^{+}(x) \cdot \mu_{\mathcal{H}\left(e_{i}\right)}^{+}(x)\right]+\left[\mu_{\mathcal{G}\left(e_{i}\right)}^{-}(x) \cdot \mu_{\mathcal{H}\left(e_{i}\right)}^{-}(x)\right]\right]}{\sum_{i=1}^{n}\left[\left[1-\sqrt{\left[\left(1-\mu_{\mathcal{G}\left(e_{i}\right)}^{2+}(x)\right) \cdot\left(1-\mu_{\mathcal{H}\left(e_{i}\right)}^{2+}(x)\right)\right]}\right]+\left[1-\sqrt{\left[\left(1-\mu_{\mathcal{G}\left(e_{i}\right)}^{2-}(x)\right) \cdot\left(1-\mu_{\mathcal{H}\left(e_{i}\right)}^{2-}(x)\right)\right]}\right]\right.}
\end{gathered}
$$

Clearly

$$
\eta_{\mathcal{F}(e)}^{2+}(x) \geq \eta_{\mathcal{G}(e)}^{2+}(x) \geq \eta_{\mathcal{H}(e)}^{2+}(x) \text { and } \eta_{\mathcal{F}(e)}^{2-}(x) \geq \eta_{\mathcal{G}(e)}^{2-}(x) \geq \eta_{\mathcal{H}(e)}^{2-}(x)
$$

Thus,

$$
\left[\eta_{\mathcal{F}(e)}^{2+}(x)-\eta_{\mathcal{H}(e)}^{2+}(x)\right] \geq\left[\eta_{\mathcal{G}(e)}^{2+}(x)-\eta_{\mathcal{H}(e)}^{2+}(x)\right] \text { and }\left[\eta_{\mathcal{F}(e)}^{2-}(x)-\eta_{\mathcal{H}(e)}^{2-}(x)\right] \geq\left[\eta_{\mathcal{G}(e)}^{2-}(x)-\eta_{\mathcal{H}(e)}^{2-}(x)\right]
$$

Hence

$$
\begin{aligned}
& \sum_{i=1}^{n}\left[\left|\eta_{\mathcal{F}\left(e_{i}\right)}^{2+}(x)-\eta_{\mathcal{H}\left(e_{i}\right)}^{2+}(x)\right|+\left|\eta_{\mathcal{F}\left(e_{i}\right)}^{2-}(x)-\eta_{\mathcal{H}\left(e_{i}\right)}^{2-}(x)\right|\right] \\
& \geq \sum_{i=1}^{n}\left[\left|\eta_{\mathcal{G}\left(e_{i}\right)}^{2+}(x)-\eta_{\mathcal{H}\left(e_{i}\right)}^{2+}(x)\right|+\left|\eta_{\mathcal{G}\left(e_{i}\right)}^{2-}(x)-\eta_{\mathcal{H}\left(e_{i}\right)}^{2-}(x)\right|\right] .
\end{aligned}
$$

Also

$$
\left[\eta_{\mathcal{F}(e)}^{2+}(x) \cdot \eta_{\mathcal{H}(e)}^{2+}(x)\right] \geq\left[\eta_{\mathcal{G}(e)}^{2+}(x) \cdot \eta_{\mathcal{H}(e)}^{2+}(x)\right] \text { and }\left[\eta_{\mathcal{F}(e)}^{2-}(x) \cdot \eta_{\mathcal{H}(e)}^{2-}(x)\right] \geq\left[\eta_{\mathcal{G}(e)}^{2-}(x) \cdot \eta_{\mathcal{H}(e)}^{2-}(x)\right]
$$

Hence

$$
\begin{aligned}
& \sum_{i=1}^{n}\left[\left[1+\left[\eta_{\mathcal{F}\left(e_{i}\right)}^{2+}(x) \cdot \eta_{\mathcal{H}\left(e_{i}\right)}^{2+}(x)\right]\right]+\left[1+\left[\eta_{\mathcal{F}\left(e_{i}\right)}^{2-}(x) \cdot \eta_{\mathcal{H}\left(e_{i}\right)}^{2-}(x)\right]\right]\right] \\
& \geq \sum_{i=1}^{n}\left[\left[1+\left[\eta_{\mathcal{G}\left(e_{i}\right)}^{2+}(x) \cdot \eta_{\mathcal{H}\left(e_{i}\right)}^{2+}(x)\right]\right]+\left[1+\left[\eta_{\mathcal{G}\left(e_{i}\right)}^{2-}(x) \cdot \eta_{\mathcal{H}\left(e_{i}\right)}^{2-}(x)\right]\right] .\right.
\end{aligned}
$$

Dividing (6) by (7), we get

$$
\frac{\sum_{i=1}^{n}\left[\left|\eta_{\mathcal{F}\left(e_{i}\right)}^{2+}(x)-\eta_{\mathcal{H}\left(e_{i}\right)}^{2+}(x)\right|+\left|\eta_{\mathcal{F}\left(e_{i}\right)}^{2-}(x)-\eta_{\mathcal{H}\left(e_{i}\right)}^{2-}(x)\right|\right]}{\sum_{i=1}^{n}\left[\left[1+\left[\eta_{\mathcal{F}\left(e_{i}\right)}^{2+}(x) \cdot \eta_{\mathcal{H}\left(e_{i}\right)}^{2+}(x)\right]\right]+\left[1+\left[\eta_{\mathcal{F}\left(e_{i}\right)}^{2-}(x) \cdot \eta_{\mathcal{H}\left(e_{i}\right)}^{2-}(x)\right]\right]\right]} \geq \frac{\sum_{i=1}^{n}\left[\left|\eta_{\mathcal{G}\left(e_{i}\right)}^{2+}(x)-\eta_{\mathcal{H}\left(e_{i}\right)}^{2+}(x)\right|+\left|\eta_{\mathcal{G}\left(e_{i}\right)}^{2-}(x)-\eta_{\mathcal{H}\left(e_{i}\right)}^{2-}(x)\right|\right]}{\sum_{i=1}^{n}\left[\left[1+\left[\eta_{\mathcal{G}\left(e_{i}\right)}^{2+}(x) \cdot \eta_{\mathcal{H}\left(e_{i}\right)}^{2+}(x)\right]\right]+\left[1+\left[\eta_{\mathcal{G}\left(e_{i}\right)}^{2-}(x) \cdot \eta_{\mathcal{H}\left(e_{i}\right)}^{2-}(x)\right]\right]\right]}
$$


and

$$
\begin{gathered}
\sqrt{1-\frac{\sum_{i=1}^{n}\left[\left|\eta_{\mathcal{F}\left(e_{i}\right)}^{2+}(x)-\eta_{\mathcal{H}\left(e_{i}\right)}^{2+}(x)\right|+\left|\eta_{\mathcal{F}\left(e_{i}\right)}^{2-}(x)-\eta_{\mathcal{H}\left(e_{i}\right)}^{2-}(x)\right|\right]}{\sum_{i=1}^{n}\left[\left[1+\left[\eta_{\mathcal{F}\left(e_{i}\right)}^{2+}(x) \cdot \eta_{\mathcal{H}\left(e_{i}\right)}^{2+}(x)\right]\right]+\left[1+\left[\eta_{\mathcal{F}\left(e_{i}\right)}^{2-}(x) \cdot \eta_{\mathcal{H}\left(e_{i}\right)}^{2-}(x)\right]\right]\right]}} \\
\leq \sqrt{1-\frac{\sum_{i=1}^{n}\left[\left|\eta_{\mathcal{G}\left(e_{i}\right)}^{2+}(x)-\eta_{\mathcal{H}\left(e_{i}\right)}^{2+}(x)\right|+\left|\eta_{\mathcal{G}\left(e_{i}\right)}^{2-}(x)-\eta_{\mathcal{H}\left(e_{i}\right)}^{2-}(x)\right|\right]}{\sum_{i=1}^{n}\left[\left[1+\left[\eta_{\mathcal{G}\left(e_{i}\right)}^{2+}(x) \cdot \eta_{\mathcal{H}\left(e_{i}\right)}^{2+}(x)\right]\right]+\left[1+\left[\eta_{\mathcal{G}\left(e_{i}\right)}^{2-}(x) \cdot \eta_{\mathcal{H}\left(e_{i}\right)}^{2-}(x)\right]\right]\right]}} .
\end{gathered}
$$

Adding (5)and (8) and divided by 2, we get

$$
\Phi^{\mathcal{B}}(\mathcal{F}, \mathcal{H}) \leq \Phi^{\mathcal{B}}(\mathcal{G}, \mathcal{H})
$$

Clearly $\alpha_{i} \leq \beta_{i} \leq \gamma_{i}$, where

$$
\begin{aligned}
& \alpha_{i}=\frac{\mu_{p\left(e_{i}\right)}^{2+}(x)+\mu_{p\left(e_{i}\right)}^{2-}(x)}{\left[\mu_{p\left(e_{i}\right)}^{2+}(x)+\eta_{p\left(e_{i}\right)}^{2+}(x)\right]+\left[\mu_{p\left(e_{i}\right)}^{2-}(x)+\eta_{p\left(e_{i}\right)}^{2-}(x)\right]^{2}}, \\
& \beta_{i}=\frac{\mu_{q\left(e_{i}\right)}^{2+}(x)+\mu_{q\left(e_{i}\right)}^{2-}(x)}{\left[\mu_{q\left(e_{i}\right)}^{2+}(x)+\eta_{q\left(e_{i}\right)}^{2+}(x)\right]+\left[\mu_{q\left(e_{i}\right)}^{2-}(x)+\eta_{q\left(e_{i}\right)}^{2-}(x)\right]^{2}}, \\
& \gamma_{i}=\frac{\mu_{r\left(e_{i}\right)}^{2+}(x)+\mu_{r\left(e_{i}\right)}^{2-}(x)}{\left[\mu_{r\left(e_{i}\right)}^{2+}(x)+\eta_{r\left(e_{i}\right)}^{2+}(x)\right]+\left[\mu_{r\left(e_{i}\right)}^{2-}(x)+\eta_{r\left(e_{i}\right)}^{2-}(x)\right]} .
\end{aligned}
$$

Clearly, $\alpha_{i}-\gamma_{i} \leq \beta_{i}-\gamma_{i}$. Since $\alpha_{i}, \beta_{i}, \gamma_{i}$ are numerical values, thus

$$
\left|\beta_{i}-\gamma_{i}\right| \leq\left|\alpha_{i}-\gamma_{i}\right| \Longrightarrow-\left|\alpha_{i}-\gamma_{i}\right| \leq-\left|\beta_{i}-\gamma_{i}\right| .
$$

Now, since

$$
\left|\alpha_{i}+\gamma_{i}\right| \leq\left|\beta_{i}+\gamma_{i}\right|
$$

Dividing (10) by (11), we get

$$
\frac{-\left|\alpha_{i}-\gamma_{i}\right|}{\left|\alpha_{i}+\gamma_{i}\right|} \leq \frac{-\left|\beta_{i}-\gamma_{i}\right|}{\left|\beta_{i}+\gamma_{i}\right|} \Longrightarrow 1-\frac{\left|\alpha_{i}-\gamma_{i}\right|}{\left|\alpha_{i}+\gamma_{i}\right|} \leq 1-\frac{\left|\beta_{i}-\gamma_{i}\right|}{\left|\beta_{i}+\gamma_{i}\right|} .
$$

Hence

$$
\Psi^{\mathcal{B}}(p, r) \leq \Psi^{\mathcal{B}}(q, r)
$$

Multiplying (9) with (12), we get

$$
\Phi^{\mathcal{B}}(\mathcal{F}, \mathcal{H}) \cdot \Psi^{\mathcal{B}}(p, r) \leq \Phi^{\mathcal{B}}(\mathcal{G}, \mathcal{H}) \cdot \Psi^{\mathcal{B}}(q, r) .
$$

Hence $\operatorname{Sim}\left(\mathcal{F}_{p}^{\mathcal{B}}, \mathcal{H}_{r}^{\mathcal{B}}\right) \leq \operatorname{Sim}\left(\mathcal{G}_{q}^{\mathcal{B}}, \mathcal{H}_{r}^{\mathcal{B}}\right)$. This proves (iv). 
Example 5. To calculate the similarity measure between the two PPBFSSs, $\mathcal{F}_{p}^{\mathcal{B}}$ and $\mathcal{G}_{q}^{\mathcal{B}}$, we choose the first sample of $\mathcal{F}_{p}^{\mathcal{B}}$ from Example 2 and $\mathcal{G}_{q}^{\mathcal{B}}$ from Example 4. $E=\left\{e_{1}, e_{2}, e_{3}\right\}$ can be defined as below (Tables 1 and 2):

Table 1

\begin{tabular}{c|c|c|c}
$\mathcal{G}_{9}^{\mathcal{B}}(e)$ & $e_{1}$ & $e_{2}$ & $e_{3}$ \\
\hline $\mathcal{F}(e)$ & $(0.6,0.7,-0.3,-0.8)$ & $(0.9,0.4,-0.7,-0.5)$ & $(0.8,0.5,-0.2,-0.9)$ \\
\hline$p(e)$ & $(0.6,0.5,-0.8,-0.3)$ & $(0.8,0.3,-0.6,-0.5)$ & $(0.7,0.4,-0.8,-0.6)$
\end{tabular}

Table 2

\begin{tabular}{c|c|c|c}
$\mathcal{G}_{q}^{\mathcal{B}}(e)$ & $e_{1}$ & $e_{2}$ & $e_{3}$ \\
\hline $\mathcal{G}(e)$ & $(0.3,0.4,-0.2,-0.3)$ & $(0.4,0.5,-0.6,-0.2)$ & $(0.6,0.2,-0.1,-0.4)$ \\
\hline$q(e)$ & $(0.5,0.4,-0.3,-0.1)$ & $(0.6,0.2,-0.4,-0.2)$ & $(0.4,0.3,-0.5,-0.6)$
\end{tabular}

Now,

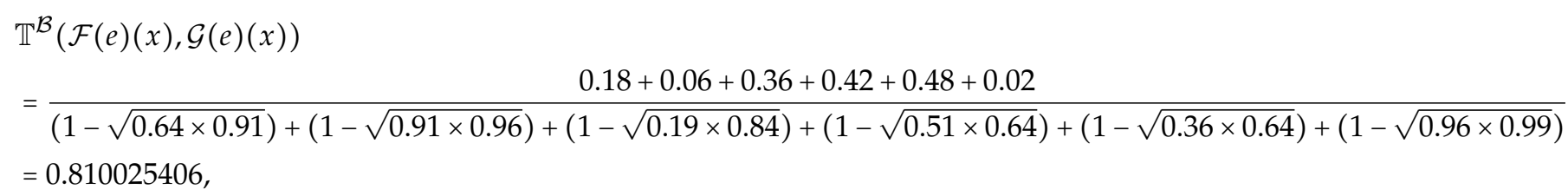

$$
\begin{aligned}
\mathbb{S}^{\mathcal{B}}(\mathcal{F}(e)(x), \mathcal{G}(e)(x)) & =\sqrt{1-\frac{2.04}{6.3256}}=0.823104458, \\
\Phi^{\mathcal{B}}(\mathcal{F}, \mathcal{G}) & =\frac{0.810025406+0.823104458}{2}=0.816564932, \\
\Psi^{\mathcal{B}}(p, q) & =1-\frac{0.408104299}{4.18746331}=0.902541403 .
\end{aligned}
$$

Hence,

$$
\operatorname{Sim}\left(\mathcal{F}_{p}^{\mathcal{B}}, \mathcal{G}_{q}^{\mathcal{B}}\right)=0.816564932 \times 0.902541403=0.736983659 .
$$

\section{Application of PPBFSS using soft model}

For the selection of school teaching education, the evaluation of teaching education is carried out according to various standards of experts. We identify a factor for the parental decision making: Academic Factor - divided into five identified elements namely Class room size, Fee system, Quality, Environment and Student/Teacher relationship. Our goal is to select the optimal one out of a great number of alternatives based on the assessment of experts against the criteria.

\subsection{Algorithm}

The algorithm for the selection of the best choice is given as:

1. Input the PPBFSS $\mathcal{F}_{p}^{\mathcal{B}}$ in tabular form.

2. Input the set of choice parameters $A \subseteq E$.

3. Compute the values of $\mathbb{T}^{\mathcal{B}}$ and $\mathbb{S}^{\mathcal{B}}$.

4. Calculate the $\Phi^{\mathcal{B}}$ value by taking $\frac{\mathbb{T}^{\mathcal{B}}+\mathbb{S}^{\mathcal{B}}}{2}$.

5. Determine the value $\Psi^{\mathcal{B}}=1-\frac{\sum\left|\alpha_{i}-\beta_{i}\right|}{\sum\left|\alpha_{i}+\beta_{i}\right|}$ for $1 \leq i \leq 5$.

6. Compute the similarity measure by taking the product of $\Phi^{\mathcal{B}}$ and $\Psi^{\mathcal{B}}$.

7. Determine maximum similarity, where maximum similarity $=\max \left\{\right.$ similarity $\left.^{i}\right\}$ for $1 \leq i \leq 5$.

8. Finally, decision is to choose as the best solution to the problem. 


\subsection{Survey study}

A parent intends to choose the popular school education source. Here we intends to choose five schools. The score of the school education source evaluated by the experts is represented by $E=\left\{e_{1}\right.$ :Class room size, $e_{2}:$ Fee system, $e_{3}:$ Quality, $e_{4}$ :Environment, $e_{5}:$ Student/Teacher relationship\}.

Table 3. PPBFSS for the ideal school education source

\begin{tabular}{c|c|c|c|c|c}
$\mathcal{L}_{p}^{\mathcal{B}}(e)$ & $e_{1}$ & $e_{2}$ & $e_{3}$ & $e_{4}$ & $e_{5}$ \\
\hline $\mathcal{L}(e)$ & $(0.9,0.2,-0.9,-0.3)$ & $(0.8,0.3,-0.7,-0.4)$ & $(0.9,0.4,-0.8,-0.3)$ & $(0.8,0.2,-0.9,-0.4)$ & $(0.9,0.3,-0.7,-0.4)$ \\
\hline$p(e)$ & $(1,0,-1,0)$ & $(1,0,-1,0)$ & $(1,0,-1,0)$ & $(1,0,-1,0)$ & $(1,0,-1,0)$
\end{tabular}

Table 4. PPBFSS for the first school education source.

\begin{tabular}{c|c|c|c|c|c}
$\mathcal{A}_{p_{1}}^{\mathcal{B}}(e)$ & $e_{1}$ & $e_{2}$ & $e_{3}$ & $e_{4}$ & $e_{5}$ \\
\hline $\mathcal{A}(e)$ & $(0.8,0.4,-0.6,-0.8)$ & $(0.5,0.7,-0.6,-0.7)$ & $(0.8,0.6,-0.7,-0.4)$ & $(0.4,0.6,-0.8,-0.5)$ & $(0.7,0.5,-0.4,-0.6)$ \\
\hline$p_{1}(e)$ & $(0.9,0.2,-0.6,-0.5)$ & $(0.8,0.3,-0.7,-0.6)$ & $(0.7,0.6,-0.5,-0.8)$ & $(0.5,0.7,-0.8,-0.4)$ & $(0.5,0.8,-0.6,-0.7)$
\end{tabular}

Table 5. PPBFSS for the second school education source.

\begin{tabular}{c|c|c|c|c|c}
$\mathcal{B}_{p_{2}}^{\mathcal{B}}(e)$ & $e_{1}$ & $e_{2}$ & $e_{3}$ & $e_{4}$ & $e_{5}$ \\
\hline$\widetilde{\mathcal{B}}(e)$ & $(0.6,0.7,-0.8,-0.4)$ & $(0.5,0.6,-0.5,-0.7)$ & $(0.7,0.6,-0.5,-0.6)$ & $(0.6,0.7,-0.8,-0.5)$ & $(0.8,0.5,-0.6,-0.7)$ \\
\hline$p_{2}(e)$ & $(0.8,0.4,-0.5,-0.8)$ & $(0.6,0.7,-0.8,-0.6)$ & $(0.4,0.6,-0.7,-0.4)$ & $(0.7,0.6,-0.5,-0.7)$ & $(0.9,0.3,-0.4,-0.8)$
\end{tabular}

Table 6. PPBFSS for the third school education source.

\begin{tabular}{c|c|c|c|c|c}
$\mathcal{C}_{p_{3}}^{\mathcal{B}}(e)$ & $e_{1}$ & $e_{2}$ & $e_{3}$ & $e_{4}$ & $e_{5}$ \\
\hline $\mathcal{C}(e)$ & $(0.8,0.4,-0.6,-0.4)$ & $(0.6,0.8,-0.5,-0.6)$ & $(0.5,0.6,-0.8,-0.4)$ & $(0.6,0.7,-0.7,-0.5)$ & $(0.7,0.6,-0.5,-0.6)$ \\
\hline$p_{3}(e)$ & $(0.6,0.8,-0.7,-0.5)$ & $(0.5,0.7,-0.6,-0.8)$ & $(0.7,0.6,-0.5,-0.6)$ & $(0.8,0.5,-0.3,-0.7)$ & $(0.5,0.8,-0.4,-0.8)$
\end{tabular}

Table 7. PPBFSS for the fourth school education source.

\begin{tabular}{c|c|c|c|c|c}
$\mathcal{D}_{p_{4}}^{\mathcal{B}}(e)$ & $e_{1}$ & $e_{2}$ & $e_{3}$ & $e_{4}$ & $e_{5}$ \\
\hline $\mathcal{D}(e)$ & $(0.6,0.7,-0.9,-0.5)$ & $(0.8,0.6,-0.6,-0.7)$ & $(0.6,0.8,-0.5,-0.6)$ & $(0.7,0.4,-0.8,-0.5)$ & $(0.5,0.6,-0.6,-0.7)$ \\
\hline$p_{4}(e)$ & $(0.9,0.3,-0.7,-0.5)$ & $(0.8,0.4,-0.6,-0.8)$ & $(0.7,0.4,-0.5,-0.6)$ & $(0.6,0.7,-0.7,-0.5)$ & $(0.9,0.3,-0.6,-0.4)$
\end{tabular}

Table 8. PPBFSS for the fifth school education source.

\begin{tabular}{c|c|c|c|c|c}
$\mathcal{E}_{p_{5}}^{\mathcal{B}}(e)$ & $e_{1}$ & $e_{2}$ & $e_{3}$ & $e_{4}$ & $e_{5}$ \\
\hline $\mathcal{E}(e)$ & $(0.6,0.8,-0.8,-0.4)$ & $(0.6,0.7,-0.6,-0.5)$ & $(0.8,0.5,-0.7,-0.6)$ & $(0.8,0.4,-0.7,-0.5)$ & $(0.9,0.3,-0.6,-0.5)$ \\
\hline$p_{5}(e)$ & $(0.7,0.5,-0.7,-0.6)$ & $(0.5,0.6,-0.8,-0.4)$ & $(0.6,0.8,-0.5,-0.7)$ & $(0.7,0.4,-0.8,-0.5)$ & $(0.8,0.5,-0.6,-0.4)$
\end{tabular}

Suppose that decision makers in the school education can provide the PBFN values for the ideal school education source, which reflect the pursuit of the ideal qualities of the school education source. The evaluations of the school education source as per PPBFSS are shown as Tables 4-8. The PBFNs values in Tables 4-8 are provided by the experts, depending on their assessment of the alternatives against the criteria under consideration. In this example, in order to find the school education source which is closest to the ideal school education source, we should calculate the similarity measure of PPBFSSs in Tables 4-8 with the one in Table 3 based on Definition 15. The threshold of the similarity should rely on the school source. Calculating the similarity measure for the five schools education source is given below the Table 9 .

From the above results, we find that the fifth school education source is closest to the ideal school education source with the highest value of the similarity measure is 0.704541488 . 
Table 9. Similarity measure for the five schools education

\begin{tabular}{c|c|c|c|c|c} 
& $\mathbb{T}^{\mathcal{B}}$ & $\mathbb{S}^{\mathcal{B}}$ & $\Phi^{\mathcal{B}}$ & $\Psi^{\mathcal{B}}$ & Similarity \\
\hline$(\mathcal{L}, \mathcal{A})$ & 0.910563838 & 0.87477231 & 0.892668074 & 0.729407489 & 0.651118778 \\
\hline$(\mathcal{L}, \mathcal{B})$ & 0.920226297 & 0.864848554 & 0.892537425 & 0.694298847 & 0.619687705 \\
\hline$(\mathcal{L}, \mathcal{C})$ & 0.90351808 & 0.886377252 & 0.894947666 & 0.588593685 & 0.526760544 \\
\hline$(\mathcal{L}, \mathcal{D})$ & 0.913193334 & 0.85720406 & 0.885198697 & 0.794320031 & 0.703131057 \\
\hline$(\mathcal{L}, \mathcal{E})$ & 0.955626388 & 0.907274181 & 0.931450284 & 0.75639194 & $\mathbf{0 . 7 0 4 5 4 1 4 8 8}$
\end{tabular}

\section{Comparison of PPBFSS approach with PBFSS}

\subsection{Algorithm}

The algorithm for the selection of the best choice is given as:

1. Input the PBFSS $\mathcal{F}_{p}^{\mathcal{B}}$ in tabular form.

2. Input the set of choice parameters $A \subseteq E$.

3. Compute the values of $\mathbb{T}^{\mathcal{B}}$ and $\mathbb{S}^{\mathcal{B}}$.

4. Calculate the similarity by taking $\frac{\mathbb{T}^{\mathcal{B}}+\mathbb{S}^{\mathcal{B}}}{2}$.

5. Determine maximum similarity, where maximum similarity $=\max \left\{\right.$ similarity $\left.^{i}\right\}$ for $1 \leq i \leq 5$.

6. Finally, make decision to choose the best solution of the problem.

We investigated the above mentioned survey study using the PBFSS approach to consider the effect of the possibility parameter. Calculating the similarity measure for the five schools education property as follows (Table 10: From the above results, the parameter has a significant impact on the calculation of the similarity

\section{Table 10}

\begin{tabular}{c|c|c|c} 
& $\mathbb{T}^{\mathcal{B}}$ & $\mathbb{S}^{\mathcal{B}}$ & Similarity \\
\hline$(\mathcal{L}, \mathcal{A})$ & 0.910563838 & 0.87477231 & 0.892668074 \\
\hline$(\mathcal{L}, \mathcal{B})$ & 0.920226297 & 0.864848554 & 0.892537425 \\
\hline$(\mathcal{L}, \mathcal{C})$ & 0.90351808 & 0.886377252 & 0.894947666 \\
\hline$(\mathcal{L}, \mathcal{D})$ & 0.913193334 & 0.85720406 & 0.885198697 \\
\hline$(\mathcal{L}, \mathcal{E})$ & 0.955626388 & 0.907274181 & 0.931450284
\end{tabular}

measure of PPBFSSs. It is observed that the first, second, third and fourth school education source from the perspective of similarity measure are quite away from the ideal school education source. If the school education source chooses the threshold 0.60 in ( $e_{2}$ fifth school), we should choose the fifth school education source as a potential school. On the contrary, when using PBFSS approach without the generalization parameter, we can not distinguish which school education source is the best one. So the possibility parameter has an important influence to the similarity measure of the fifth school education source.

\section{Conclusion}

PPBFSS approach is more scientific and reasonable than PBFSS approach without the generalization parameter in the process of decision-making. This work presented a PPBFSS to solve the phenomena related to decision making. Moreover, we discussed some operational properties namely complement, union, intersection and find similarity measure. So in future, we should consider the possibility Pythagorean cubic and spherical soft set theory.

Acknowledgments: The author is thankful to the reviewers for their numerous and significant suggestions that raised the consistency of the ideas presented in this paper.

Conflicts of Interest: "The author declares no conflict of interest."

\section{References}

[1] Zadeh, L. A. (1965). Fuzzy sets. Information and Control, 8(3), 338-353.

[2] Atanassov, K. (1986). Intuitionistic fuzzy sets. Fuzzy Sets E Systems, 20(1), 87-96. 
[3] Lee, K. M. (2000). Bipolar-valued fuzzy sets and their operations. In Proceding of International Conference on Intelligent Technologies, Bangkok, Thailand, 2000 (pp. 307-312).

[4] Yager, R. R. (2013). Pythagorean membership grades in multicriteria decision making. IEEE Transactions on Fuzzy Systems, 22(4), 958-965.

[5] Molodtsov, D. (1999). Soft set theory First results. Computers and Mathematics with Applications, 37, 19-31.

[6] Maji, P. K., Biswas, R., \& Roy, A. R. (2001). Fuzzy soft set. Journal of Fuzzy Mathematics, 9(3), 589-602.

[7] Maji, P. K., Biswas, R., \& Roy, A. R. (2001). On intuitionistic fuzzy soft set. Journal of Fuzzy Mathematics, 9(3), 677-692.

[8] Abdullah, S., Aslam, M., \& Ullah, K. (2014). Bipolar fuzzy soft sets and its applications in decision making problem. Journal of Intelligent $\mathcal{E}$ Fuzzy Systems, 27(2), 729-742.

[9] Alkhazaleh, S., Salleh, A. R., \& Hassan, N. (2011). Possibility fuzzy soft set. Advances in Decision Sciences, 2011, Article ID 479756, https:/ /doi.org/10.1155/2011/479756.

[10] Peng, X. D., Yang, Y., Song, J., \& Jiang, Y. (2015). Pythagorean fuzzy soft set and its application. Computer Engineering, 41(7), 224-229.

[11] Yager, R. R., \& Abbasov, A. M. (2013). Pythagorean membership grades, complex numbers, and decision making. International Journal of Intelligent Systems, 28(5), 436-452.

[12] Mohana, K., \& Jansi, R. (2018). Bipolar Pythagorean fuzzy sets and their application based on multi-criteria decision-making problems. International Journal of Research Advent in Technology, 6, 3754-3764.

[13] Akram, M., \& Ali, G. (2020). Hybrid models for decision-making based on rough Pythagorean fuzzy bipolar soft information. Granular Computing, 5(1), 1-15.

[14] Alkhazaleh, S., \& Salleh, A. R. (2012). Generalised interval-valued fuzzy soft set. Journal of Applied Mathematics, 2012, Article ID 870504, https:/ / doi.org/10.1155/2012/870504.

[15] Chen, T. Y. (2018). An interval-valued Pythagorean fuzzy compromise approach with correlation-based closeness indices for multiple-criteria decision analysis of bridge construction methods. Complexity, 2018, Article ID 6463039, https:/ / doi.org/10.1155/2018/6463039.

[16] Jana, C., Senapati, T., \& Pal, M. (2019). Pythagorean fuzzy Dombi aggregation operators and its applications in multiple attribute decision-making. International Journal of Intelligent Systems, 34(9), 2019-2038.

[17] Jana, C., \& Pal, M. (2018). Application of bipolar intuitionistic fuzzy soft sets in decision making problem. International Journal of Fuzzy System Applications, 7(3), 32-55.

[18] Karaaslan, F. (2017). Possibility neutrosophic soft sets and PNS-decision making method. Applied Soft Computing, 54, 403-414.

[19] Peng, X., \& Yang, Y. (2016). Fundamental properties of interval-valued Pythagorean fuzzy aggregation operators. International Journal of Intelligent Systems, 31(5), 444-487.

[20] Zhang, X. (2016). Multicriteria Pythagorean fuzzy decision analysis: A hierarchical QUALIFLEX approach with the closeness index-based ranking methods. Information Sciences, 330, 104-124. 